\title{
Idiopathic osteonecrosis of the medial tibial plateau
}

J. R. Valenti

J. A. Illescas
A. Barriga
R. Dölz

\begin{abstract}
Osteonecrosis of the medial tibial plateau is characterized by acute pain on the medial aspect of the knee. Progression can lead to articular collapse and requires early diagnosis and treatment. We studied seven patients affected of idiopathic osteonecrosis of the tibial plateau. The mean age was 62 years and the mean follow-up 42 months. We performed roentgenograms in all patients, bone scans in three patients and magnetic resonance image (MRI) in five. MRI shows T1-weighted low-intensity signal and T2weighted high-intensity signal with a surrounding area of intermediate low-intensity signal. An increased focal uptake was seen at bone scan. Histological findings showed necrotic bone with empty lacunae. Surgical treatment consisted of tibial subchondral drilling in four patients - two of them by failure of conservative treatment, and a total knee arthroplasty in other two. One patient had a satisfactory evolution with conservative treatment. Idiopathic osteonecrosis of the tibial plateau must be considered in elderly patients with knee pain over the medial tibial plateau. At early stages, decompression with tibial drilling must be considered. This procedure allows a prompt and effective relief of symptoms.
\end{abstract}

\section{KEYWORDS}

Osteonecrosis; Idiopathic tibial medial plateau; Drilling

Correspondence: J. R. Valentí

Department of Orthopaedics and Traumatology

University Clinic, School of Medicine, University of Navarra.

Pío XII, 36, 31008 Pamplona, Spain

E-mail: jrvalenti@unav.es

Tel.: +34-948-255400

Fax: +34-948-296500 


\section{INTRODUCTION}

Osteonecrosis of the medial tibial plateau is an analogous pathology to the necrosis of the medial femoral condyle and was first described by D'Anglejan and colleagues in 1976 [4]. This is an unusual lesion characterized by a sudden onset (without traumatic precedent) of acute pain on the medial aspect of the knee [3, 4, 6-8, 12]. This lesion appears in patients more than 60 years old, and it is more frequent in women. It is characterized by intense pain, mainly at night, and in-creases with physical activity. Physical examination shows intense pain with palpation of the medial part of the tibial plateau, without any other interesting findings $[3,4,6,10,15]$. The initial roentgenograms may be normal, or show evidence of incipient osteoarthritis, common among the elderly. At this initial stage, technetium radionuclide scans and magnetic resonance image (MRI) confirm our diagnostic suspicion [3, 4, 7, 10]. We present our study concerning the natural history of this pathology, its diagnosis and treatment.

\section{MATERIALS AND METHODS}

We evaluated seven patients (two male and five female) affected by idiopathic osteonecrosis of the medial tibial plateau. We reviewed their medical records and imaging studies, and diagnosis was confirmed with histological analysis in patients who underwent surgery. We evaluated the patients' age at presentation, sex, associated pathologies, alcohol usage (abuse being defined as $>400 \mathrm{ml} \%$ of ethanol per week), presentation symptoms, family history, weight, involvement of other joints and use of corticosteroids. Patients affected by secondary or post-traumatic osteonecrosis were excluded. All knees were included in our radiographic evaluation with standard anteroposterior and lateral projections, establishing initial stage and evolution. Radiographic sequential stages were obtained on the basis of Ficat and Arlet classification modified for the knee (Table 1) $[13,18]$. Technetium ${ }^{-99 m}$ bone scintigraphy was performed in three knees and MRI in five. Diagnosis of osteonecrosis was confirmed with histological analysis in six patients.

\section{RESULTS}

The mean age of these patients was 62 years (range 53- 74 years). The left knee was involved in four patients, and the right knee in three (Table 2). Mean follow-up was 42 months (range 2-5 years). All patients had acute and intense knee pain, mainly at night. Five patients $(71.4 \%)$ presented mild effusion, and six patients $(85.7 \%)$ intense pain with palpation of the anterior aspect of the medial tibial plateau. Slight restriction of range of motion was observed: mean flexion was $100^{\circ}$ (range $90-110^{\circ}$ ), and maximum extension varied between 0 to $-10^{\circ}$. No signs of instability were observed in five of these knees. Routine laboratory studies revealed normal findings. None of the patients had a traumatic precedent, rheumatologic pathologies, sickle-cell disease, alcoholism or chronic use of corticosteroids.

Three patients (42.8\%) showed stage I roentgenograms (Fig. 1), two patients at stage III and two patients at stage IV. Stage II was not observed. Technetium ${ }^{-99 m}$ bone scans were obtained in three of seven knees, showing an increased up-take in the subchondral region of the medial tibial plateau (Fig. 2). MRI was obtained in five patients; three with 
previous bone scan and two patients just MRI, showing the characteristic pattern of low signal intensity in T1-weighted (Fig. 3) and T2-weighted (Fig. 4) images.

Three patients (42.8\%) were submitted to initial non-operative treatment with restricted weight-bearing associated with non-steroidal anti-inflammatory drugs. Two of them required further operation (arthroscopic decompression by subchondral drilling) due to failure of conservative treatment, showing a good evolution during the monitoring. Four patients (57.1\%) were submitted to decompression by drilling (Fig. 5), two stage I and two stage III patients at initial roentgenograms (the stage III patients for failure of nonoperative treatment), with good evolution and relief of symptoms. Histological findings showed necrotic bone with empty lacunae and fatty degeneration (Fig. 6). Two patients (28.5\%) presented with stage IV roentgenograms and underwent a total knee replacement (Fig. 7).

\section{DISCUSSION}

Necrosis of the medial tibial plateau has a prevalence of $2 \%$ in big series, with $80 \%$ of the patients being women more than 55 years old [12]. The etiology is unknown, and at this present time a variety of factors may con-tribute to the clinical picture $[4,7,8,12$, $14,18]$. Some authors think that necrosis, just like true infarction in bone, results from vascular insufficiency [8, 16]. Trauma is another possible etiology. Subchondral microfractures may produce a partial microscopic collapse of the tibial plateau $[10,13$, 18]. Eventual bone collapse may be the consequence of large lesions of the tibial plateau that involve subchondral bone, with development of radio-lucent areas with a sclerotic halo [10, 18]. Osteoporotic bone in elderly women may be a risk factor in relation to microfractures in the subchondral tibial plateau. The histological findings of Narváez et al. [14] suggest that the etiology of spontaneous osteonecrosis results from an insufficient subchondral fracture in an osteoporotic bone [8]. Spontaneous necrosis of the knee is also described as an arthroscopy complication $[2,5,18]$.

In some occasions, this pathology is not recognized even by specialists, who don't diagnose this lesion until collapse on femoral or tibial osteoarthritis is set. Onset of acute and sudden pain without traumatic precedent, with more inflammatory than mechanical characteristics, must help us to suspect this pathology. Pain is frequently related to an unusual increase of activity or to a mild traumatism, and the symptoms occur mainly at night, associated to local hypersensitivity and edema [4, 6-9, 13, 14]. Physical examination shows intense pain in the medial side of the tibial plateau, usually close to the joint line of the knee $[7,8,13,14]$.

Differential diagnosis is established in comparison with meniscal pathology, femoropatellar arthritis, pes anserinus tendinitis and transitional osteoporosis, in view of the fact that there are two important clinical characteristics that distinguish this lesion from other knee pathologies. The first is night pain. Patients with degenerative articular or meniscal lesions have worsening of symptoms with activity and improvement with rest. The second is that the pain location is under the joint line, on the medial aspect of the tibial plateau.

Roentgenograms may be normal at initial stages, or present subchondral radiolucent areas if the process is advanced. Bone scan and magnetic resonance help to confirm diagnosis at an early stage, which in patients with symptoms indicative of the condition 
allows us to undertake early treatment, preventing development of the pathology. Technetium $^{-99 \mathrm{~m}}$ bone scans show an increased focal up-take in the medial part of the tibial plateau. In MRI, a focal area of low signal in the affected tibial plateau is seen. Fat suppression in T2-weighted images presents a typical zone of high signal intensity (bone marrow edema) with a low signal intensity area in the lesion's center. These findings are similar to femoral condyle necrosis $[1,2,5]$.

The first three patients of our series showed normal or almost normal radiographs; at that time, due to a poor knowledge of this pathology we performed bone scans, and in those cases with a positive result, completed the study with MRI. In the last two patients of the series we only performed MRI. If the articular collapse was evident (stage IV of Ficat and Arlet) no complementary studies were necessary. At the present time we confirm the diagnosis only with MRI.

Histological analysis shows necrotic bone, surrounded by an area of fibrovascular granulation tissue and focal areas of osteoclastic and macrophagic activity [8]. Yamamoto and Bullough [18] set a typical image of subchondral fracture of the medial tibial plateau, with a focal area of necrosis on trabecular bone. In some patients, onset of symptoms is consecutive to progressive collapse of the articular surface. The clinical course varies according to the radiographic stage. If radiographs are negative for bone collapse, the lesion may bring about a remission, and patients may improve their symptomatology between 3 and 12 months. Owing to the fact that many patients have initial osteoarthritis changes, the knee cannot be completely asymptomatic. In other patients with radiographic evidence of sclerotic lesions and small areas of collapse, progression can lead to the total collapse of the tibial plateau, generally requiring surgery.

Recognition of the problem is the most important consideration before initiating treatment. Two patients of our series were diagnosed later (more than 6 months after the onset of symptoms) and that affected treatment and prognosis.

If the lesion is not extensive, therapeutic programs focused on decreasing the weightbearing associated to non-steroidal anti-inflammatory drugs may prevent collapse of the medial tibial plateau. Intra-articular steroid injections can relieve pain, but they are not recommended [12]. Arthroscopic debridement has been performed, but its efficacy has not been evaluated. Core decompression of the necrotic zones, as is done in the hip, is the treatment of choice for patients with lesions in stage I and stage II, because it allows a rapid and effective relief of symptoms [17]. At initial stages, we have obtained excellent results with arthroscopy and percutaneous drilling made from outside to inside and proximal to distal. The number of subchondral drillings depends on the size of the lesion. Some authors recommend hemiarthroplasty for patients who do not improve with conservative treatment, and with radiographic evidence of collapse on the medial side but a well-preserved lateral compartment [7, 11]. Knee arthroplasty is the only choice for the treatment of stage IV lesions, and this procedure has also been used when other treatment regimens have failed.

Finally, we must suspect this pathology of unknown etiology, the typical patient being a woman more than 55 years old with acute and nocturnal pain in the medial side of the knee. Initial roentgenograms are often normal or without visible alterations, or may show a radiolucent area in the subchondral bone and MRI confirms diagnosis. Early recognition of this problem is essential for a satisfactory treatment. 


\section{REFERENCES}

1) Bjorkengren $\mathrm{A}$, Al Rowaih $\mathrm{A}$, Lind-strand A, Wingstrand $\mathrm{H}$, Thorngren $\mathrm{K}$, Pettersson H (1990) Spontaneous osteonecrosis of the knee: value of MR imaging in determining prognosis. AJR Am J Roentgenol 154:331-336.

2) Brahme S, Fox J, Ferkel R, Friedman M, Flannigan B, Resnick D (1991) Osteonecrosis of the knee after arthroscopic surgery: diagnosis with MR imaging. Radiology 178:851-853.

3) Carpintero-Benitez P, Collantes-Estevez E (1998) Osteonecrosis of the tibial plateau. Clin Rheumatol 17:95-98.

4) Ecker M, Lotke P (1995) Osteonecrosis of the medial part of the tibial plateau. J Bone Joint Surg Am 77:596-601.

5) Johnson T, Evans J, Gilley J, De Lee J (2000) Osteonecrosis of the knee after arthroscopic surgery for meniscal tears and chondral lesions. Arthroscopy 16:254-261.

6) Le L, Savy J, Orcel P, Liote F, Kuntz D, Tubiana J, Kaplan G, Laredo J (1999) Osteonecrosis-like syndrome of the medial tibial plateau can be due to a stress fracture. MR findings in 13 patients. Rev Rheum Engl 66:323-330.

7) Lotke P, Ecker M (1988) Osteonecrosis of the knee: current concepts review. J Bone Joint Surg Am 70:470-473.

8) Lotke P, Ecker M, Alavi A (1997) Painful knees in older patients. J Bone Joint Surg Am 59:617-621.

9) Lotke P, Ecker M, Barth P, Lonner J (2000) Subchondral magnetic resonance imaging in early osteoartrosis associated with tibial osteonecrosis. Arthroscopy 16(1):76-81.

10) Marmor L (1998) Fracture as a com-plication of osteonecrosis of the tibial plateau. A case report. J Bone Joint Surg Am 70:454-457.

11) Marti C, Rodriguez M, Zanetti M, Romero J (2000) Spontaneous osteonecrosis of the medial compartment of the knee: a MRI follow-up after conservative and operative treatments, preliminary results. Knee Surg Sports Traumatol Arthrosc 8:83-88.

12) Mont M, Jones L, La Porte D (1999) Symptomatic multifocal osteonecrosis: a multicenter study. Clin Orthop 369:312-326.

13) Mont M, Baumgarten K, Rifai A, Bluemke D, Jones L, Hungerford D (2000) Atraumatic osteonecrosis of the knee. J Bone Joint Surg Am 82:1279- 1290.

14) Narváez J, Narváez JA, Rodriguez-Moreno J, Roig-Escofet D (2000) Osteonecrosis of the knee: differences among idiopathic and secondary types. Rheumatology 39:982-989.

15) Patel D, Breazeale N, Behr C, Warren R, Wickiewicz T, O’Brien S (1998) Osteonecrosis of the knee: current clinical concepts. Knee Surg Sports Traumatol Arthrosc 6:2-11.

16) Rudberg U, Ahlbäck S, Uden R, Rydberg J (1993) Radiocolloid uptake in spontaneous osteonecrosis of the knee: a case report. Clin Orthop 287:25-29.

17) Valenti J, Leyes M, Schweitzer D (1998) Spontaneous osteonecrosis of the knee: treatment and evolution. Knee Surg Sports Traumatol Arthrosc 6(1):12-15.

18) Yamamoto $T$, Bullough $P$ (2000) Spontaneous osteonecrosis of the knee: the result of subchondral insufficiency fracture. J Bone Joint Surg Am 82:858- 866. 


\begin{tabular}{|l|l|}
\hline \multicolumn{2}{|c|}{ Table 1. Ficat and Arlet classification modified for the knee } \\
\hline Stage I & Normal degenerative changes in X-ray \\
\hline Stage II & Cystic or radiolucent areas in subchondral bone \\
\hline Stage III & Crescent sign or subchondral collapse with development of sclerotic halos \\
\hline Stage IV & $\begin{array}{l}\text { Arthritic changes (narrowing of articular space, marginal osteophytes, } \\
\text { destruction of cartilage and tibial and femoral articular collapse) }\end{array}$ \\
\hline
\end{tabular}

Table 2. Patient evaluation data. F female, $M$ male, L left, R right, NAS narrowing of articular space, AC articular collapse, BS bone scan, MRI magnetic resonance imaging.

\begin{tabular}{|c|c|c|c|c|c|c|c|}
\hline Case & Age & Sex & Side & X-ray & BS & MRI & Treatment \\
\hline 1 & 71 & F & L & NAS & Yes & Yes & Arthroscopy and drilling \\
\hline 2 & 54 & F & R & NAS & No & Yes & Arthroscopy and drilling \\
\hline 3 & 73 & F & L & AC & No & No & TKA \\
\hline 4 & 54 & M & R & NAS & Yes & Yes & Arthroscopy and drilling \\
\hline 5 & 53 & M & R & NAS & No & Yes & Conservative \\
\hline 6 & 74 & F & L & AC & No & No & TKA \\
\hline 7 & 54 & F & R & NAS & Yes & Yes & Arthroscopy and drilling \\
\hline
\end{tabular}




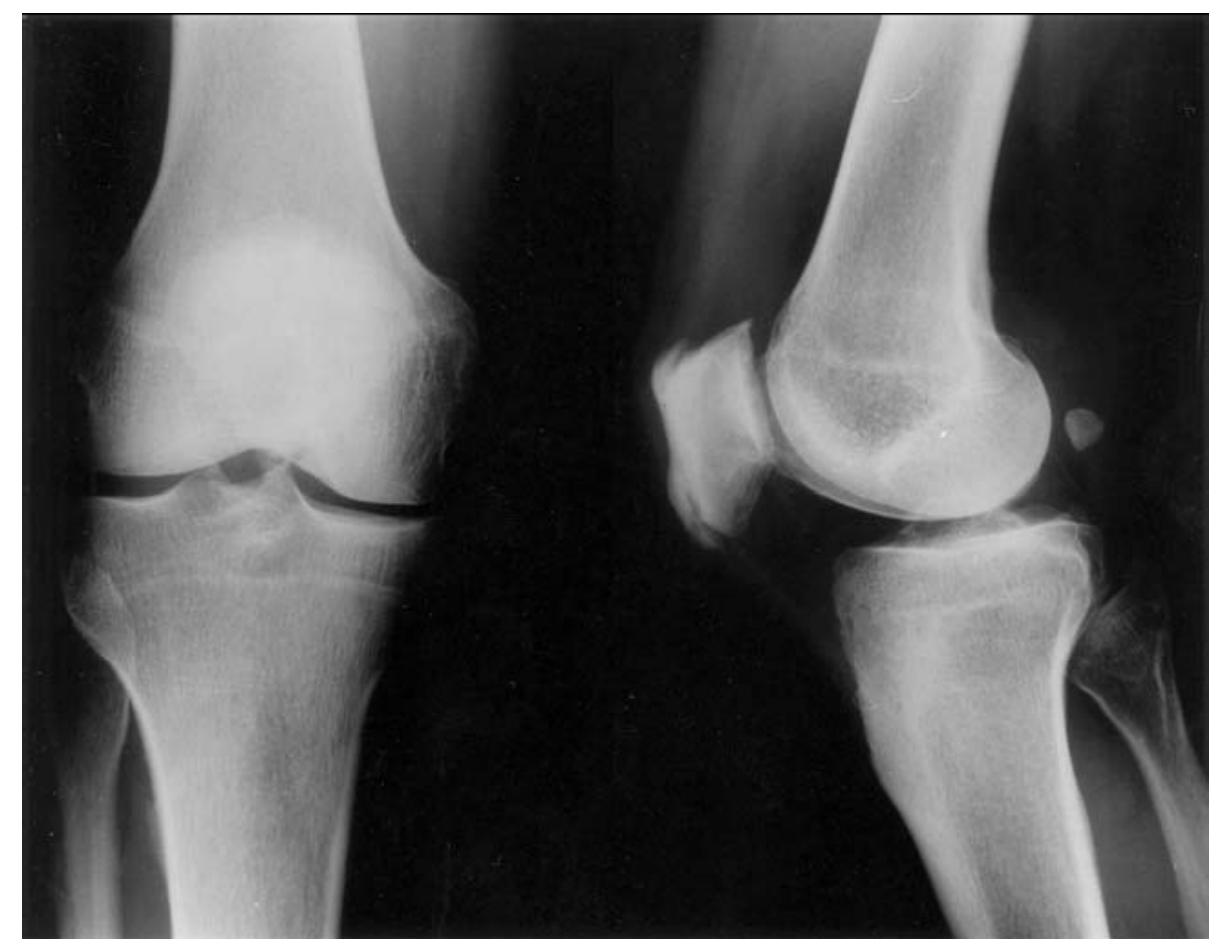

Figure 1. A 54-year-old man: X-ray with normal degenerative changes

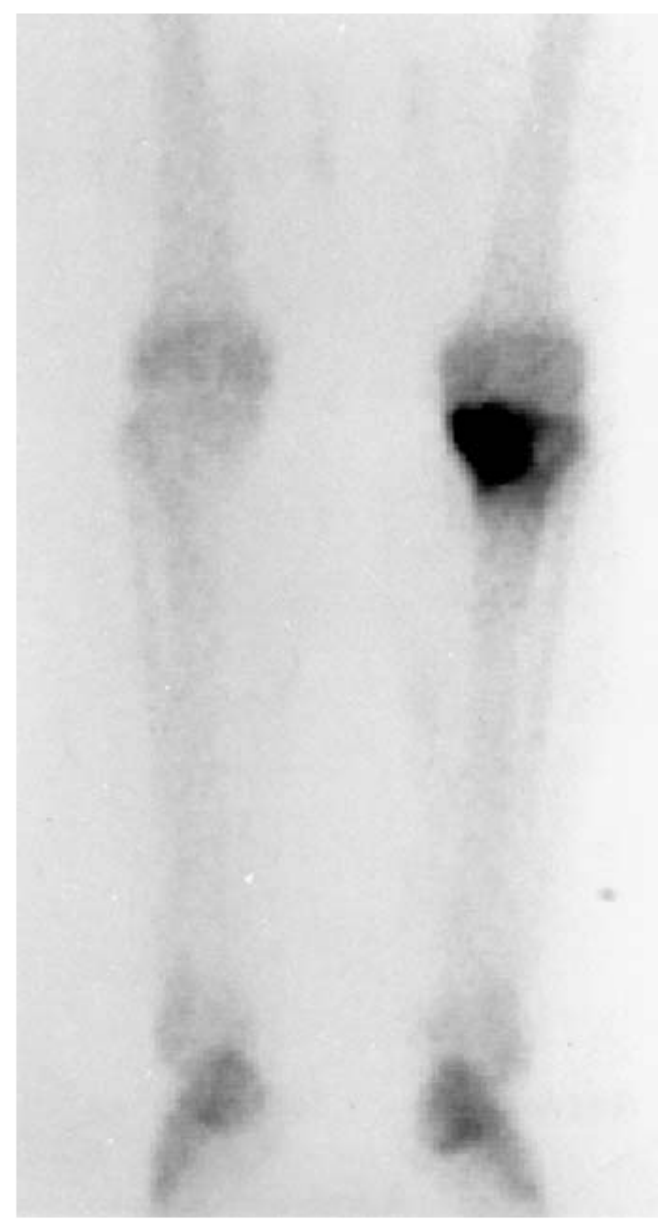

Figure 2. Bone scintigraphy with Tc99m shows focal increase uptake in the tibial plateau (same patient as in Fig. 1) 


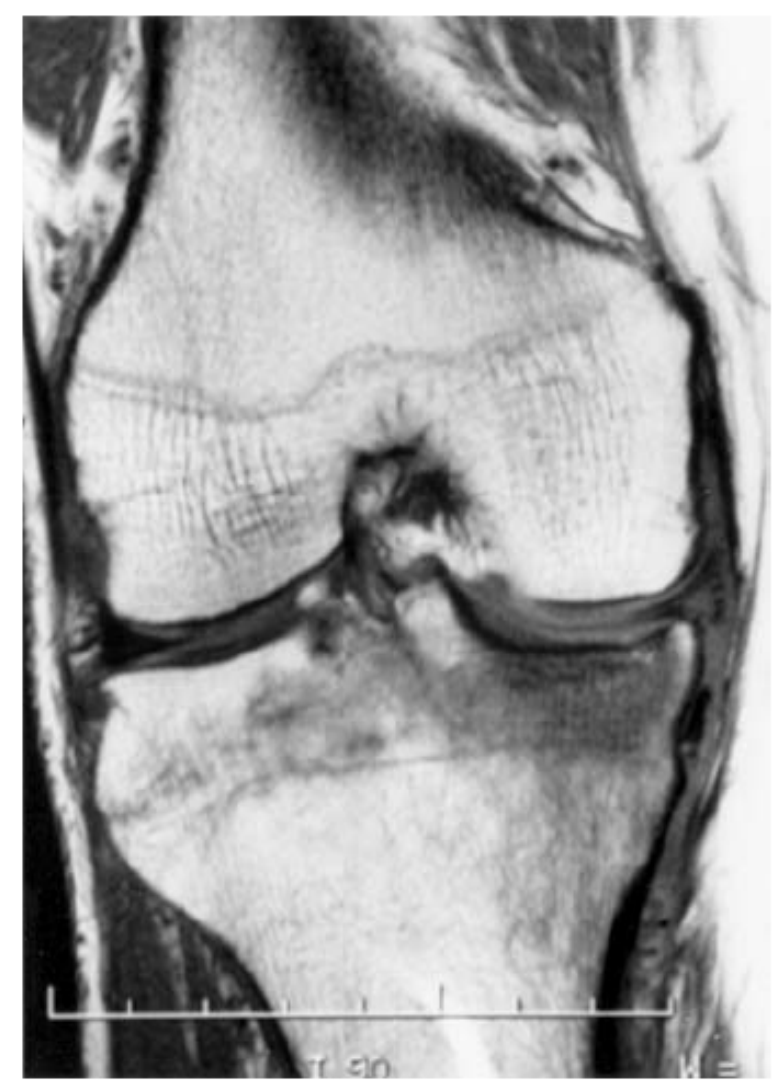

Figure 3. Magnetic resonance images show T1-weighted low-intensity signal (same patient as in Fig. 1)

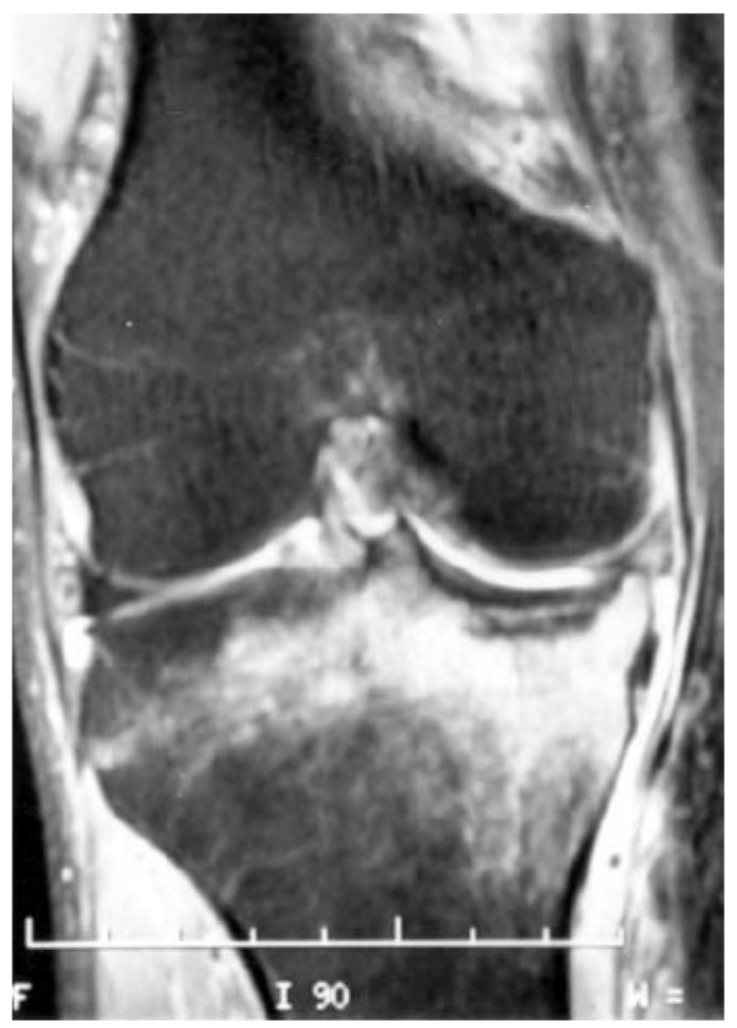

Figure 4. T2-weighted high-intensity signal (narrowing edema) with surrounding area of intermediate low-intensity signal (same patient as in Fig. 1) 


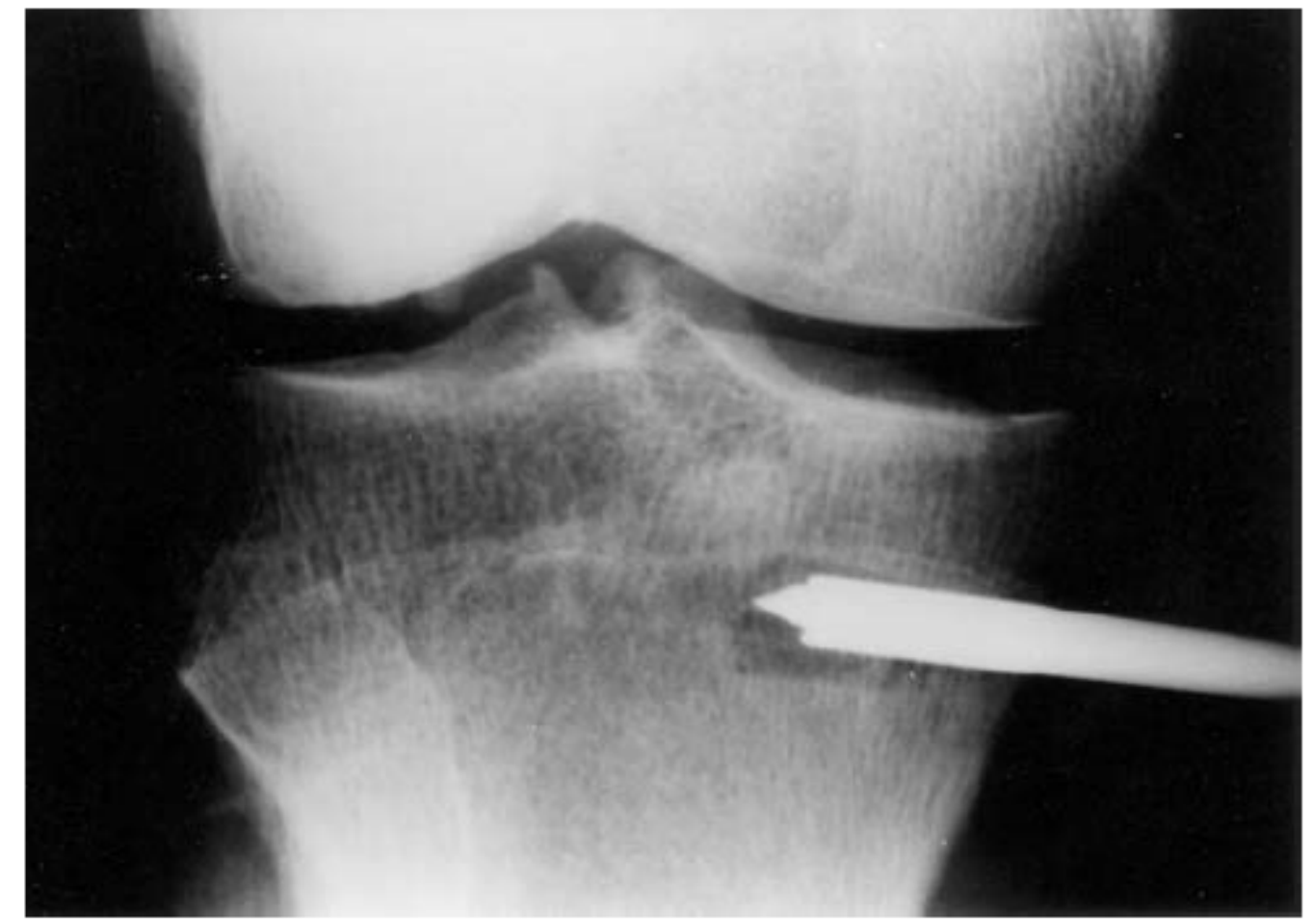

Figure 5. Biopsy of bone (same patient as in Fig. 1)

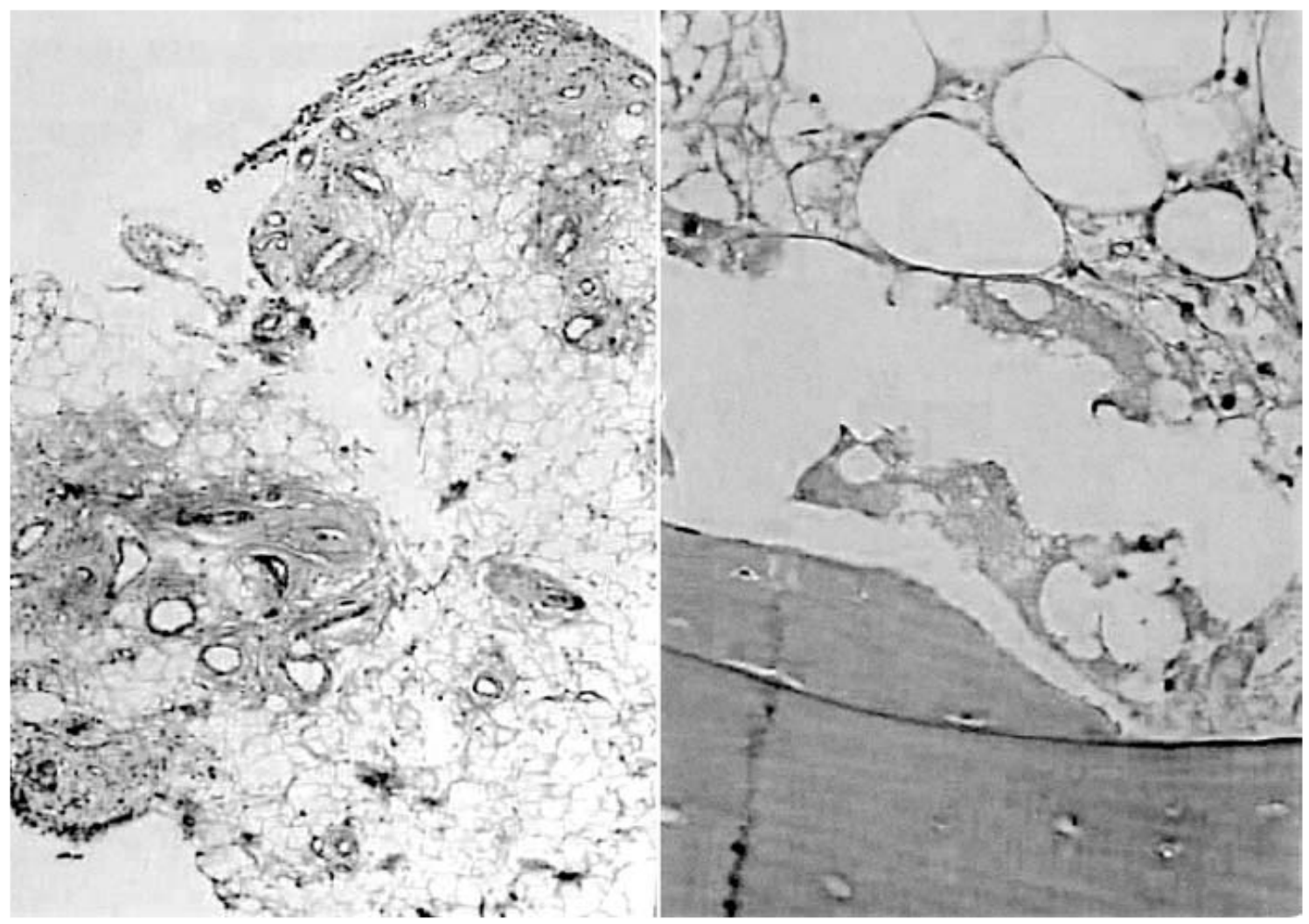

Figure 6. Histological findings reflect necrotic bone with empty lacunae (same patient as in Fig. 1) 


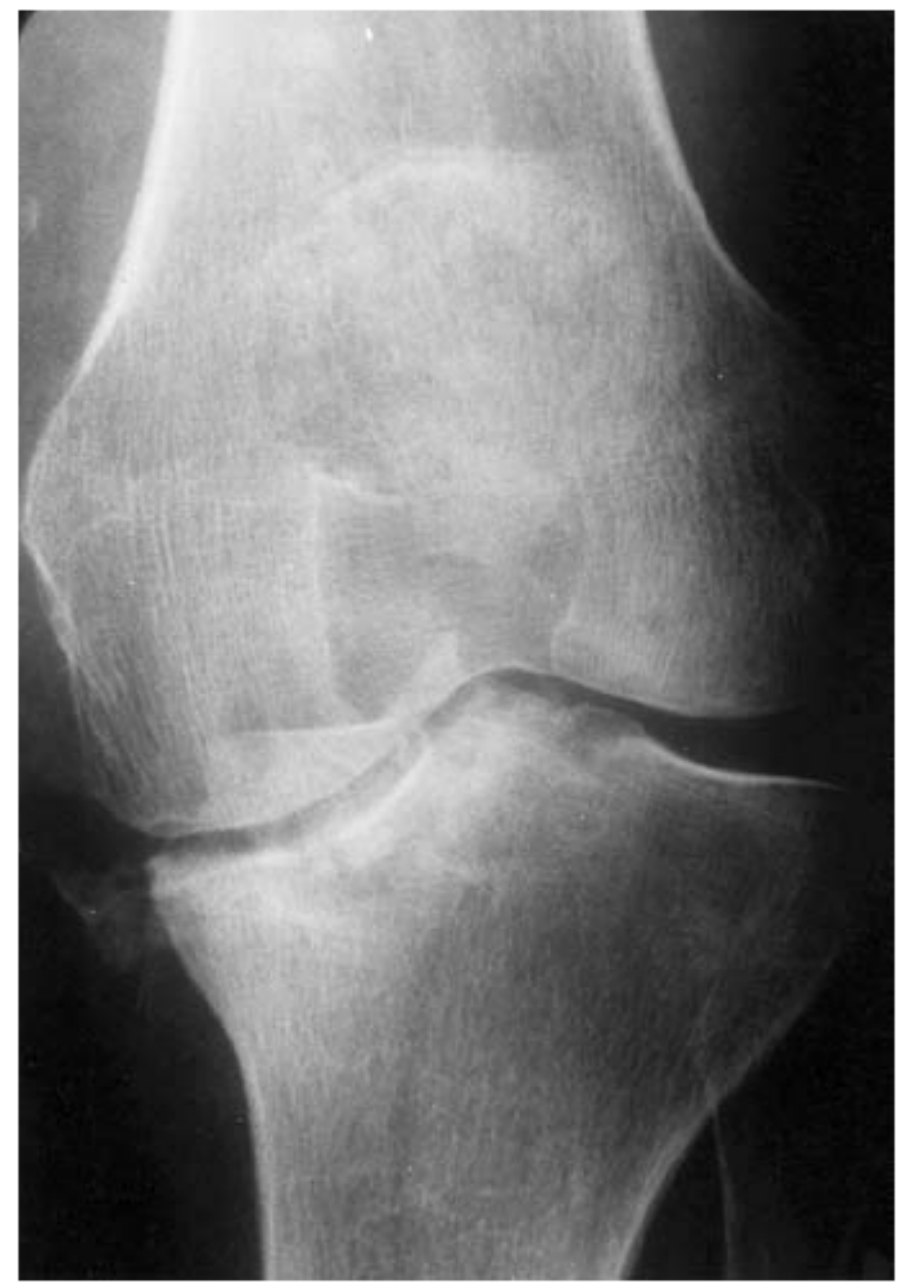

Figure 7. X-ray showing medial tibial plateau collapse of the osteonecrotic bone at stage IV. 